\title{
From blanket quarantine in Wuhan to distant centralized quarantine in Shijiazhuang: the evolution of China's COVID-19 quarantine approach
}

\author{
Baona Wang ${ }^{1}$ (1) $\cdot$ Hui Zheng ${ }^{1}$
}

Received: 7 February 2021 / Accepted: 28 April 2021 / Published online: 7 May 2021

(c) Springer-Verlag GmbH Germany, part of Springer Nature 2021

\begin{abstract}
With COVID-19 spreading globally, the World Health Organization (WHO) declared a pandemic on March 11, 2020. COVID-19 swept many countries and regions worldwide. An effective response to COVID-19 requires newer and more creative tools. In this paper, we discussed the evolution of China's COVID-19 quarantine approach, compared the blanket quarantine in Wuhan and the distant centralized quarantine in rural areas of Shijiazhuang, and analyzed the important issues which authorities will have to pay attention to ensure success from the moment they begin to take close contacts to the single room isolation in a distant quarantine center. The large-scale distant centralized quarantine strategy in Shijiazhuang cut off the transmission of COVID-19 within 1 month. This strategy may inform other countries and regions of a feasible and effective approach to combat the global pandemic of COVID-19.
\end{abstract}

Keywords COVID-19 · Quarantine strategy $\cdot$ Single room isolation $\cdot$ Rural areas

With COVID-19 spreading globally, the World Health Organization (WHO) declared a pandemic on March 11, 2020. Early isolation of airborne infection is an effective way to control the spread of the disease [1,2]. During the early stages of the pandemic, intrusive actions including enforced quarantine helped cut the spread of COVID-19 and have saved both lives and livelihoods [3]. COVID-19 is categorized as a severe acute respiratory syndrome, a quarantinable disease [4]. The beneficial effects of quarantining during an epidemic have been confirmed by studies [5, 6]. Quarantine separates and restricts the movement of people who were exposed to a contagious disease to check if they become sick.

Different types of quarantine can be utilized, including but not limited to individual quarantine, active monitoring and centralized quarantine. However, an effective response to COVID-19 requires newer and more creative tools.

Hui Zheng

zhenghui0715@hotmail.com

1 Department of Anesthesiology, National Cancer Center/ National Clinical Research Center for Cancer/Cancer Hospital, Chinese Academy of Medical Sciences and Peking Union Medical College, Beijing 100021, China
COVID-19 was first reported in early December 2019 in Wuhan, China. With the rapid transmission of COVID19, Wuhan declared a blanket quarantine on January 23, 2020, when the city was put on a complete lockdown with residents confined to their homes and transportation banned. However, without rapid diagnosis, about $80 \%$ of the cluster transmission occurred in families [7], and the risk of crossinfection in hospitals was high. On February 2, 2020, a centralized quarantine and treatment policy was implemented by the Chinese government. Centralized quarantine of patients and close contacts contributed to a reduction in COVID-19 transmission [8]. The citywide quarantine in Wuhan lasted 76 days until April 8, 2020, when the authorities determined that the transmission of COVID-19 had been brought under control.

A year later, a sudden flare-up emerged in rural areas of Shijiazhuang, the capital of North China's Hebei province. More than 300 were diagnosed with COVID-19 between January 2 and January 12, 2021. The authorities decided that they should alter their quarantine strategy as conditions in rural areas differ from those in urban centers like Wuhan city. Transmission among rural villagers is more difficult to control due to their gathering practices as well as substandard sanitation services and hygiene habits. The Chinese government decided to send all the closest contacts 
of infected persons to a purpose-built isolation station that was some distance away from their communities, where centralized medical and other services to meet daily needs were provided. The contacts were kept under observation in single-room apartments, what the British would call bedsits for between 14 and 21 days. More than 20,000 people, comprising close contacts of infected persons and also secondary close contacts (contacts of contacts) were transferred. And this distant centralized quarantine appears to have promptly reduced the spread of COVID-19. From the first infected cases reported on January 2, 2021, the COVID-19 in Shijiazhuang was effectively under control by January 27 (Fig. 1).

An analysis of the method adopted in Shijiazhuang suggests a number of advantages. It cut off the spread of COVID-19 among family members and villagers and reduced the number of new infections. The isolation of contacts made it more convenient to disinfect households in the affected villages and enabled timely diagnosis and treatment as well as targeted education about COVID-19.

But authorities who may wish to consider using this method will have to pay attention to several issues to ensure success from the moment the close contacts are rounded up and taken to the single-room isolation centers. Undoubtedly, it is important to take efficient measures to prevent the spread of COVID-19 when the transfers are taking place. In China, the contacts were fitted with surgical masks or $\mathrm{N}-95$ respirators-those worn by health professionals, and the vehicles carrying them were restricted to half capacity and one person per seat, with disinfectants always on hand. That is just the start of a 2- to 3-week journey that requires close supervision at a critical period for defeating COVID19. Other key steps are needed.

First, when people are mandatorily isolated, governments must provide facilities to meet their basic needs [4]. In Shijiazhuang, each bedsit was equipped with a bed, electric heater, television, toilet and sink, and WIFI access. More than 7000 people, including government officials, community workers and volunteers, were available to ensure that the basic needs of those in quarantine were met. Viral tests were carried out every 2 days. And medical professionals were accessible around the clock.

Second, psychological problems including boredom, stress, and sleep disturbances during the epidemic of COVID-19 must be widely attended to $[9,10]$. Psychological counseling during quarantine is extremely important. In Shijiazhuang, a 24-h hotline was open for consultation on psychological problems. Considerate services were provided for children, elders, pregnant women and people with disabilities. Guardians or personal care workers are also necessary for those unable to take care of themselves.

Third, physical health during quarantine matters. Structured indoor activity improves both physical and mental health $[11,12]$. Nutritious food was available in Shijiazhuang for everyone free of cost. Individualized meals were provided for members of special groups, for instance, diabetics, children, pregnant women, and undernourished or obese people. People with existing medical conditions were monitored and treated as necessary.

This large-scale distant centralized quarantine strategy in China promptly cut off the transmission of COVID-19

Number of cases diagnosed with COVID-19 in January in Shijiazhuang

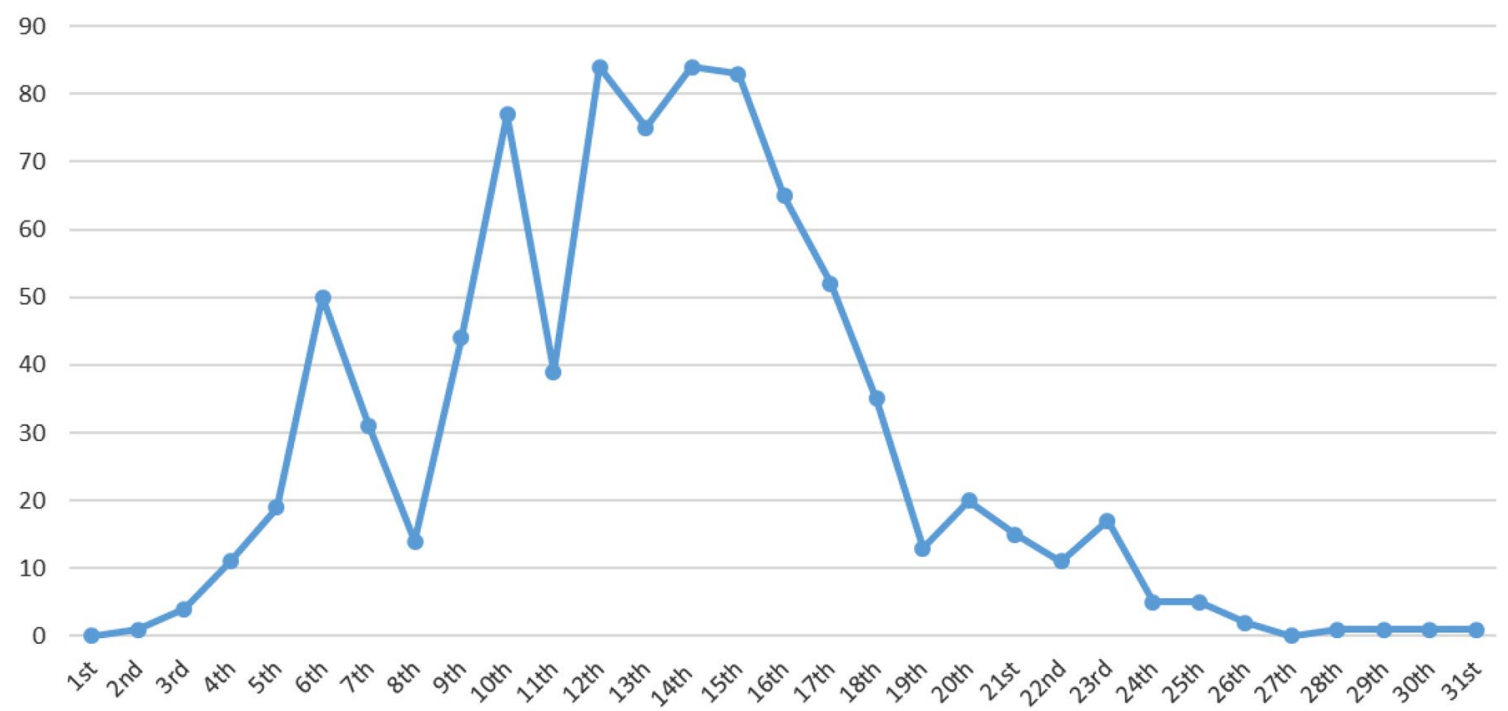

Fig. 1 Number of cases diagnosed with COVID-19 in January in Shijiazhuang. The first infected case was reported on January 2, 2021. Between January 2 and January 12, 2021, more than 300 were diag- nosed with COVID-19. Then the large-scale distant centralized quarantine was implemented. Between January 19 and January 31, the number of diagnosed cases descended to a very low level 
within 1 month, and the evidence suggests it may be especially effective in rural areas with similar characteristics. This strategy may inform other countries and regions of a feasible and effective approach in whole or part to combat the global pandemic of COVID-19.

Author contributions $\mathrm{WBN}$ and $\mathrm{ZH}$ have contributed to letter design, letter-related data analysis and letter writing.

Funding There are no funding sources to report.

\section{Declarations}

Conflict of interest The authors declare that they have no competing interests.

Ethical approval This letter to the editor does not require/warrant ethical approval from the institutional review board.

\section{References}

1. Cheng VCC, Wong SC, Chen JHK, et al. Escalating infection control response to the rapidly evolving epidemiology of the coronavirus disease 2019 (COVID-19) due to SARSCoV-2 in Hong Kong. Infect Control Hosp Epidemiol. 2020;41:493-8.

2. Zuckerman NS, Pando R, Bucris E, et al. Comprehensive analyses of SARS-CoV-2 transmission in a public health virology laboratory. Viruses. 2020;12:854.
3. Bauch CT, Anand M. COVID-19: when should quarantine be enforced? Lancet Infect Dis. 2020;20:994-5.

4. Parmet WE, Sinha MS. Covid-19-the law and limits of quarantine. N Engl J Med. 2020;382:e28.

5. Adhikari SP, Meng S, Wu Y-J, et al. Epidemiology, causes, clinical manifestation and diagnosis, prevention and control of coronavirus disease (COVID-19) during the early outbreak period: a scoping review. Infect Dis Poverty. 2020;9:29.

6. Bedford J, Enria D, Giesecke J, et al. WHO strategic and technical advisory group for infectious hazards. COVID-19: towards controlling of a pandemic. Lancet. 2020;395:1015-8.

7. Report of the WHO-China Joint Mission on Coronavirus Disease 2019. (COVID-19). https://www.who.int/docs/default-source/ coronaviruse/who-china-joint-mission-on-covid-19-final-report. pdf. Accessed 2 Mar 2020.

8. Pan A, Liu L, Wang C, et al. Association of public health interventions with the epidemiology of the COVID-19 outbreak in Wuhan. China JAMA. 2020;323:1915-23.

9. Muscogiuri G, Barrea L, Savastano S, et al. Nutritional recommendations for CoVID-19 quarantine. Euro J Clin Nutr. 2020;74:850-1.

10. Wu C, Chen X, Cai Y, et al. Risk factors associated with acute respiratory distress syndrome and death in patients with coronavirus disease 2019 pneumonia in Wuhan. China JAMA Int Med. 2020;180:934-43.

11. Gradidge PJ, Kruger HS. Physical activity, diet and quality of life during mandatory (COVID-19) quarantine following repatriation. SAGE Open Med Case Rep. 2020;8:2050313X20972508.

12. Vogel EA, Zhang JS, Peng K, et al. Physical activity and stress management during COVID-19: a longitudinal survey study. Psychol Health. 2021;1-11. 